\title{
Movement Problems of Solid ObJects IN 3D COMPUTER ANIMATION
}

\author{
Skala, T.; Jelic, A. \& MrVAC, N.
}

Abstract: Using modern methods of visualization it is possible to simulate a human locomotor system. Multimedia multi-dimensional (2D and 3D) computer programs allow realistic view of human movement. Despite the $2 D$ static content that is possible to create in a high-level of photorealistic static scene, the photorealism in $3 D$ animation is more difficult to create. This paper will be carried out theoretically in terms of research and practical verification of the results. It demonstrates the feasibility of a precise mathematical definition of human movement through animation of walk. It offers video realistic view in a way that as much as possible is closer to realistic virtual scenes.

Key words: 3D computer animation, solid objects, visualisation, movement problems
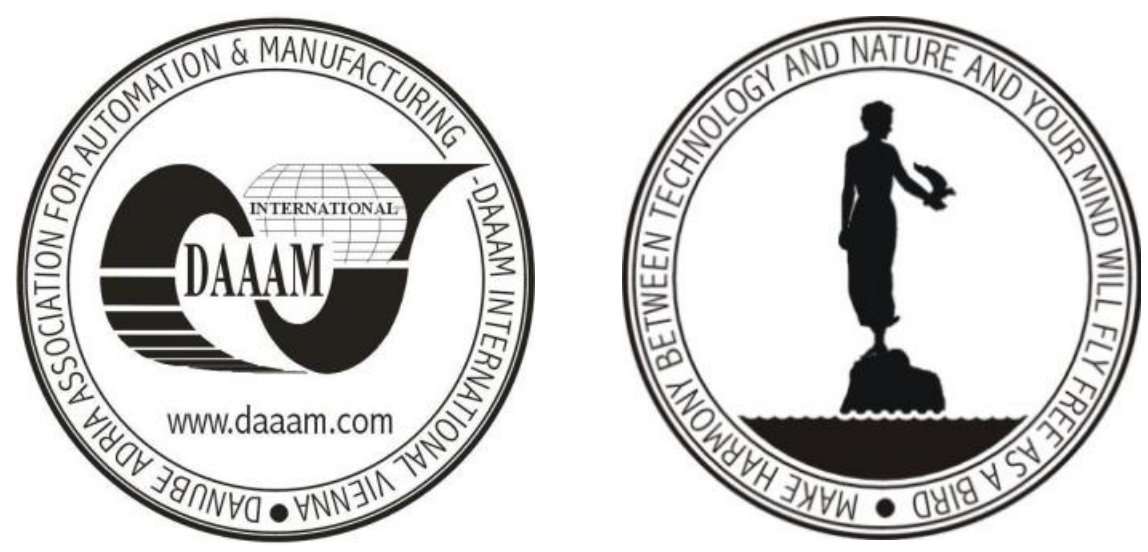

Authors' data: Dr. Sc. Skala, T[ibor]; Jelic, A[ntonija]; Univ.Prof. Dr. Sc. Mrvac, N[ikola], University of Zagreb, Faculty of Graphic Arts, Getaldiceva 2, 10000 Zagreb, Croatia, tibor.skala@grf.hr, tonkyca@gmail.com, nikola.mrvac@grf.hr

This Publication has to be referred as: Skala, T[ibor]; Jelic, A[ntonija] \& Mrvac, N[ikola] (2010). Movement Problems of Solid Objects in 3D Computer Animation, Chapter 16 in DAAAM International Scientific Book 2010, pp. 151-158, B. Katalinic (Ed.), Published by DAAAM International, ISBN 978-3-901509-74-2, ISSN 17269687, Vienna, Austria

DOI: $10.2507 /$ daaam.scibook.2010.16 
Skala, T.; Jelic, A. \& Mrvac, N.: Movement Problems of Solid Objects in 3D Com...

\section{Introduction}

Since the simulation of movement in computer graphics is quite big problem where it still is not fixed how to make it real in this work research was made on theoretical basis. Determination of the reaction of the object in interaction with external forces is the basis of achieving realistic motion of solid objects. Effect of force gives the object linear and angular acceleration with respect to the mass and weight distribution, and thereby causes a change in velocity of the object. Due to changes in speed and position of the object new forces are made, which again cause change in the movement of the object. Resulting acceleration, velocity and position changes are determined by the equations of motion of solid objects (Skala et. al. 2008). Based on these equations the state vector object and its change are formed to allow continuous renewal movement of the object. If the object moves through the space that is not completely empty, it will interact with other objects. Since the objects move independent of each other, it is necessary to establish their contacts and calculate the appropriate response to achieve an accurate simulation of interaction between two objects.

\section{Equations of motion of solid objects}

If the point $A$, whose position is in relation with point $B$ defined by vector $r(t)$ rotates, around an axis that passes through the point $B$, change the position of point $A$ is possible to express as a product of vectors of position and angular velocity (1).

$$
\begin{aligned}
\dot{r}(t) & =\omega \times r(t) \\
|\dot{r}(t)| & =|\omega||r(t)| \sin \theta
\end{aligned}
$$

Orientation of the object, $R(t)$ is possible to display as a transformed form of the local coordinate system, while the matrix columns are shown as vectors of relative position of the object. Thus, changes in the rotational matrix can be calculated by the product of angular velocity vector and each column of rotation matrix (Rigau et al. 2002).

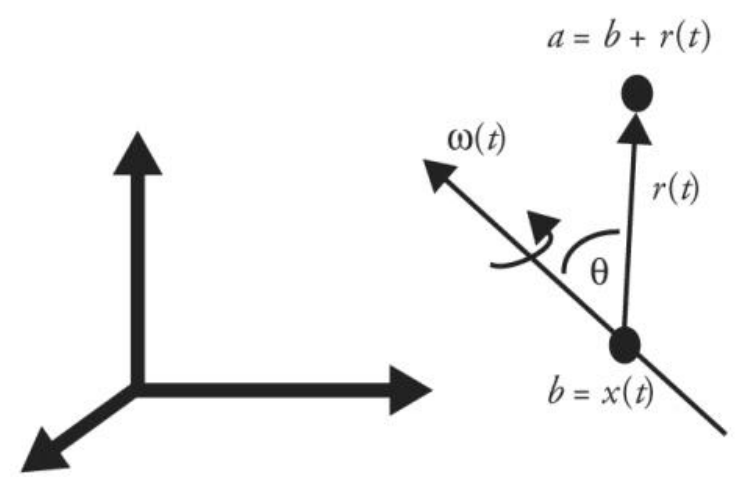

Fig. 1. Relative position of the object

$$
\begin{aligned}
R(t) & =\left[\begin{array}{lll}
R_{1}(t) & R_{2}(t) & R_{3}(t)
\end{array}\right] \\
\dot{R}(t) & =\left[\begin{array}{lll}
\omega(t) \times R_{1}(t) & \omega(t) \times R_{2}(t) & \omega(t) \times R_{3}(t)
\end{array}\right]
\end{aligned}
$$


If the matrix that represents the vector product is defined (3), it is possible to show the relation (2) as a product of matrices (4).

$$
\begin{aligned}
& A \times B=\left[\begin{array}{c}
A_{y} \cdot B_{z}-A_{z} \cdot B_{y} \\
-\left(A_{z} \cdot B_{x}\right)+A_{x} \cdot B_{z} \\
A_{x} \cdot B_{y}-A_{y} \cdot B_{x}
\end{array}\right]=\left[\begin{array}{ccc}
0 & -A_{x} & A_{y} \\
A_{z} & 0 & -A_{x} \\
-A_{y} & -A_{x} & 0
\end{array}\right]\left[\begin{array}{c}
B_{x} \\
B_{y} \\
B_{z}
\end{array}\right]=A * B \\
& \dot{R}(t)=\omega(t) * R(t)
\end{aligned}
$$

For the point $Q$ on a solid object, whose position in the local coordinate system of the object is constant and marked with $q$, position in the global coordinate system, $q(t)$, is determined by relation (5), where $x(\mathrm{t})$ denotes the position of the object in the scene. With derivation by the time it is possible to determine the equation of velocity point (Dwan, 2006) (6).

$$
\begin{gathered}
q(t)=R(t) q+x(t) \\
\dot{q}(t)=\omega(t) \times(q(t)-x(t))+v(t)
\end{gathered}
$$

As the effect of linear force $F$, at the point of mass $m$, is defined by the relation (7), and the total value of external force by the relation (8), it is possible to determine the acceleration caused by the action of the total external force, and thus the speed of a point in each time step.

$$
\begin{gathered}
F=m \cdot a \\
F(t)=\sum F_{i}(t)
\end{gathered}
$$

Since the mass distribution of an object in computer graphics is designated by certain items of mass, the centre of gravity of the object is defined by relation (9).

$$
x(t)=\frac{\sum m_{i} q_{i}(t)}{M}
$$

The effect of force on a point that is part of a solid object, affects by torque on the object by rotary equivalent linear force (10).

$$
\tau_{t}(t)=(q(t)-x(t)) \times F_{i}(t)
$$

$$
\tau(t)=\sum \tau_{t}(t)
$$

What will be the total quantity of motion of solid objects, $P(t)$, determines the value of momentum of each particle (11). For the coordinate system whose axis coincides with the main focus, equality (11) can be written as a product of the total mass of an object and its velocity (12).

$$
\begin{aligned}
& P(t)=\sum m_{i} \dot{q}_{i}(t) \\
& P(t)=M \cdot v(t)
\end{aligned}
$$

If equality (12) is derived by the time it is possible to observe that change of momentum equals the force on the object, which means that the interaction consists 
of force by an amount equal but opposite direction resulting in no change in momentum, that momentum remains preserved.

$$
\dot{P}(t)=M \cdot \dot{v}(t)=F(t)
$$

For the point mass $m$ in the object it is possible to calculate the moment of momentum with the radius-vector product and vector product of velocity and mass point. Total moment of momentum of solid object is obtained by summarizing the points of moments that make the object (14).

$$
\begin{aligned}
L(t) & =\sum\left((q(t)-x(t)) \times m_{i} \cdot(\dot{q}(t)-v(t))\right) \\
& =\sum\left(R(t) q \times m_{i} \cdot(\omega(t) \times(q(t)-x(t)))\right) \\
& =\sum\left(m_{i} \cdot(R(t) q \times(\omega(t) \times R(t) q))\right)
\end{aligned}
$$

Distribution of mass of the object in scene, inertia tensor, in the local system of the object $I_{\text {object }}$, is described by the matrix (15). The density of object points $q=\left(q_{x}, q_{y}, q_{z}\right)$ is equal to $\rho$.

$$
I_{\text {object }}=\left[\begin{array}{ccc}
I_{x x} & I_{x y} & I_{x z} \\
I_{x y} & I_{y y} & I_{y z} \\
I_{x z} & I_{y z} & I_{z z}
\end{array}\right]
$$

$I_{x x}=\iiint \rho(q) \cdot\left(q_{y}^{2}+q_{z}^{2}\right) d x d y d z$

Given the transformation of the object it is possible to transform and the inertia tensor (16).

$$
I(t)=R(t) I_{\text {object }} R(t)^{T}
$$

Object is possible to introduce with a vector containing its location, orientation, momentum and momentum of momentum (Qin et al. 2005) (17). Since the mass of the object and its inertia tensor in the local coordinate system are not values depending on the time, it is possible to establish a change of state vector under the action of force (18), which allows recovery of state vector during the time in which the force operates.

$$
\begin{aligned}
& S(t)=\left[\begin{array}{l}
x(t) \\
R(t) \\
P(t) \\
L(t)
\end{array}\right] \\
& \frac{d}{d t}=\left[\begin{array}{l}
x(t) \\
R(t) \\
P(t) \\
L(t)
\end{array}\right]=\left[\begin{array}{c}
v(t) \\
\omega(t) * R(t) \\
F(t) \\
\tau(t)
\end{array}\right]
\end{aligned}
$$

Restoring the state vector is achieved by using appropriate methods to solve differential equations formed. In the simplest implementation the Euler method is used. It is restoring the value of state vector achieved by multiplying the time derivative values at the beginning of time step size and time step. A disadvantage of 
this procedure is that the time step size affects on the accuracy, so the numerical approximated path, if time frequency is huge, significantly deviate from the ideal of continuous paths. By taking smaller steps this problemcan be solved, but that way is too demanding and to increase the accuracy the methods with greater efficiency are used. The most common is the use of Runge-Kutta methods (in particular RungeKutta fourth order) for balancing speed, accuracy and ease of use (Shalizi \& Crutchfield, 1999).

\section{Interaction of the objects}

If the particle is moving with constant speed towards the fixed plane under arbitrary angle it is possible to determine whether a collision occurred with respect to the value of equation (19) where $E(p)$ indicates a general equation of the plane, $a, b, c$ and $d$ coefficients of the general equations, and $p$ the particle that represents the position in space and has no physical characteristics.

$$
E(p)=a \cdot x+b \cdot y+c \cdot z+d
$$

Since the position of the particles is being renewed in each time step with respect to its average speed within the time frame, in every time step $t_{i}$, is necessary to check

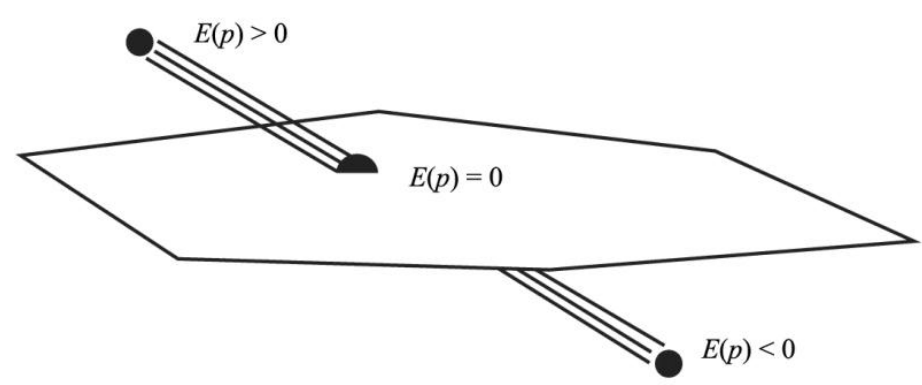

Fig.2. Intersection of convex object and plate

the position of particles relative to the plane. If the function $E\left(p\left(t_{i}\right)\right)$ for some time $t$ takes a value of zero or less than zero, which means that the current position of the particle on the plane or the back of the plane, it is possible to conclude that in the time between $t_{i-1}$ and $t_{i}$ crash was performed.

Similarly it is possible to determine the most accurate collisions of convex objects. Testing is performed for each altitudeof one object by checking the position in relation to each polygon of the other object (Schroeder \& Hanrahan, 1993). If the tested function for each polygon takes a negative value, it is considered that the top one object is inside the other (Torrance \& Sparrow, 1967).

Testing for concave objects is carried out in a way that from the observed peak straight line is constructed in a particular direction, for which is then examined the existence of intersections with the polygon object. The penetration is determined by the total number of intersections, while an odd number of intersections indicate that a peak is inside the object, and even that it is outside. In the case that the intersection of the constructed object and straight line is exactly on edge or on top of the object and if straight line is collinear with the edge of the object is very difficult to determine the 
number of intersections. The better way is to construct straight line in the other direction and re-examine.

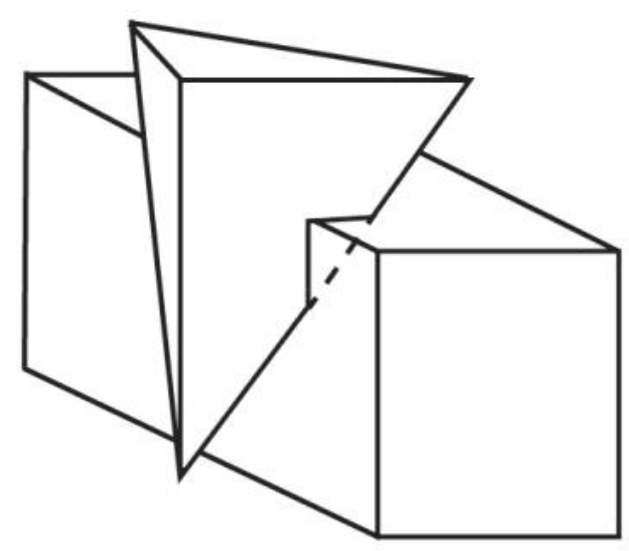

Fig.3. Penetration of two objects

Since, as shown, penetration is possible although the top of an object is not within the other; with these tests it is not possible to determine all the intersections. Therefore, in order to obtain all the intersection, examination of the intersection of the edges of a polygon and the other object is done. This test can identify all the penetration, but the price of testing is quite high, so in the most cases testing are made on the peaks.

\section{The dynamics of the collision}

In the collisions, the objects interact with the impulse $J$. It is possible to use impulses to determine the change in momentum, $\Delta P(20)$ and thus the movement of the object after the collision.

$$
J=F \cdot \Delta t
$$

$$
J=F \cdot \Delta t=M \cdot a \cdot \Delta t=M \cdot \Delta v=\Delta(M \cdot v)=\Delta P
$$

When two objects touch, the contact points are determined by relative position (21), the relative velocity in the direction of normal (22) and speed before the collision (23).

$$
\begin{gathered}
r_{A}=p_{A}-x_{A}(t) \\
r_{B}=p_{B}-x_{B}(t) \\
v_{r e l}=\left(\dot{p}_{A}(t)-\dot{p}_{B}(t)\right) \cdot n \\
\dot{p}_{A}(t)=v_{A}(t)+\omega_{A}(t) \times r_{A} \\
\dot{p}_{B}(t)=v_{B}(t)+\omega_{B}(t) \times r_{B}
\end{gathered}
$$




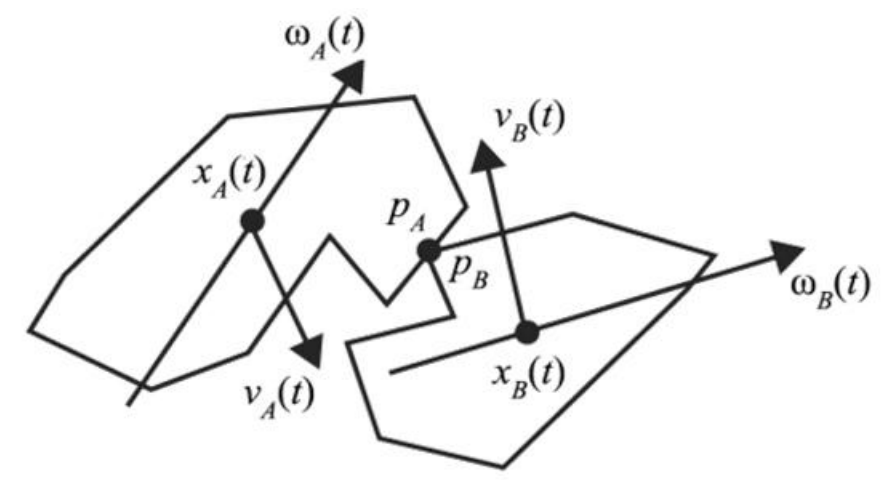

Fig. 4. Relative velocity and the speed in case when two objects touch

Given the impact of impulse $(J=j \cdot n)$ on linear and angular velocity, it is possible to form equations for linear and angular velocities of objects after the collision (24).

$$
\begin{gathered}
v_{A}^{+}=v_{A}^{-}+\frac{j \cdot n}{M_{A}} \\
v_{B}^{+}=v_{B}^{-}+\frac{j \cdot n}{M_{B}} \\
\omega_{A}^{+}=\omega_{A}^{-}+I_{A}^{-1}(t) \cdot\left(r_{A} \times j \cdot n\right) \\
\omega_{B}^{+}=\omega_{B}^{-}+I_{B}^{-1}(t) \cdot\left(r_{B} \times j \cdot n\right)
\end{gathered}
$$

Using these linear and angular velocities after the collision, it is possible to define the relative speed of points in the direction of normal after the collision (25).

$$
v_{r e l}^{+}=\left(v_{A}^{+}(t)+\omega_{A}^{+}(t) \times r_{A}-v_{B}^{+}(t)+\omega_{B}^{+}(t) \times r_{B}\right) \cdot n
$$

Since the relative velocity before and after the collisions areassociated with coefficient of elasticity (26), it is possible to calculate the amount of pulse $j$ (27).

$$
j=\frac{v_{r e l}^{+}=-\varepsilon \cdot v_{r e l}^{-}}{\frac{1}{M_{A}}+\frac{1}{M_{B}}+n \cdot\left(I_{A}^{-1}(t)\left(r_{A} \times n\right)\right) \times r_{A}+\left(I_{B}^{-1}(t)\left(r_{B} \times n\right)\right) \times r_{B}}
$$

\section{Conclusion}

In this paper, the theoretical side of the problem of movement of solid objects in 3D computer animation is considered. A detailed mathematical background is developed supported with equations in order to give as better as possible perspective of $3 \mathrm{D}$ realistic view. It has shown what happens when two objectsintersect. If the contact points get closer to each other, their relative velocity will be in the direction of normal to the plane of contact. It is possible, therefore, to determine whether in the contact collisionoccurred. If a collision is unavoidable it is necessary to determine the 
amount of pulses in order to restore the linear and angular velocity of each object. If there are multiple contact points of two objects, it is necessary to study the collision of each point and restore momentum and moment of momentum, if a collision occurred. After restoring the momentum the process is repeated to determine the existence of new contact points. Repeating the process is done until it detects no collision.

Limitations of this study can be seen in the problems of lack of strong computing resources that are needed to solve the complex mathematical equations. For this purpose, after the problem is solved and complex equations defined, the movements of solid objects in 3D computer graphics will look more realistic. In the future work it is planned to implement the qualitative analysis of machining characteristics of different resources, including a new cluster node on the Faculty of Graphic Arts where the plan is to carry out various tests and measurements of complex mathematical equations. So, the strategy is determined by future work and implementation of complex computer render procedures. A detailed analysis identifies the need of complex calculations for advanced visualization methods for large amounts of graphics and multimedia data in modern printing technology.

\section{References}

Dwan, C. (2006). Bioinformatics Benchmarks on the Dual Core Intel Xeon Processor, The BioTeam, Inc., Cambridge, MA 2006.

Qin, L.; Zhuang, Y.; Pan, Y. \& Wu, F. (2005). Video segmentation using Maximum Entropy Model, Journal of Zhejiang University SCIENCE, ISSN 1009-3095, 2005.

Rigau, J.; Feixas, M. \&Sbert, M. (2002). Entropy-based adaptive supersampling. In P. Debevec and S. Gibson, editors, The 13th Eurographics Workshop on Rendering, Poster Papers Proceedings, 63-70, Pisa, Italy, June 2002. National Research Council. Held in Pisa, Italy.

Schroeder, P. \& Hanrahan, P.(1993) On the form factor between two polygons. Computer Graphics Proceedings (Proceedings of SIGGRAPH'93), 27:163-164, August 1993. Held in Anaheim (CA), USA.

Shalizi, C. R. \& Crutchfield, J. P. (1999). Computational mechanics: Pattern and prediction, structure and simplicity. Working Paper 99-07-044, Santa Fe Insitute, Santa Fe (NM), USA, July 1999.

Skala, T.; Todorovac, M. \& Mrvac, N. (2008) Technical Analysis of Analogies of Stereo Displaying Techniques with 3D Generated Scenes in Visualitization // DAAAM International Scientific Book 2008/Katalinic, Branko (ur.). Vienna: DAAAM International, 789-796

Torrance, K. E. \& Sparrow, E. M.(1967). Theory for off-specular reflection from roughened surfaces. J. Opt. Soc. Amer., 579 :1105 1114, Sept. 1967 